International Journal of Pure and Applied Mathematics

Volume 100 No. 1 2015, 7-17

ISSN: 1311-8080 (printed version); ISSN: 1314-3395 (on-line version)

url: http://www.ijpam.eu

doi: http://dx.doi.org/10.12732/ijpam.v100i1.2

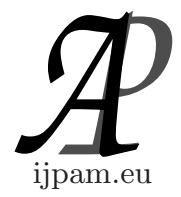

\title{
THE LAPLACE TRANSFORM OF THE HURWITZ-LERCH ZETA-FUNCTION
}

\author{
Ya. Vorobyov \\ Department of Computer Algebra and Discrete Mathematics \\ I.I. Mechnikov Odessa National University \\ St. Dvoryanskaya 2, 65026 Odessa, UKRAINE
}

\begin{abstract}
Formula for Laplace transform of product of the Hurwitz and Lerch zeta-function is obtained.
\end{abstract}

AMS Subject Classification: 11M06, 44A15

Key Words: Laplace transform, Hurwitz zeta-function, Lerch zeta-function

\section{Introduction}

Integral transforms (Laplace, Forier, Mellin) play an important role in the analytic number theory. In the works of Titchmarsh[7], Atkinson[1], Ivič[3][4], Jutila[5], Lukkarinen[6] it has been pointed out the connection between moments $I_{k}(I)$ of $\left|\zeta\left(\frac{1}{2}+i t\right)\right|^{2 k}$ on $[0, T]$ and Laplace transform $L_{k}\left(\frac{1}{2}\right)$ or modified Mellin transform $Z_{k}(\sigma+i t), k=1,2, \ldots$ Balčiunas and Laurincikas[2] investigated the Laplace transform of the Dirichlet $L$-function with principal character.

Our aim is to study the Laplace transform for product of the Hurwitz and Lerch functions.

Received: August 23, 2014

(c) 2015 Academic Publications, Ltd. url: www.acadpubl.eu 
The classical Laplace transform $L_{f}(s), s=\sigma+i t$, of the function $f(x)$ is defined by

$$
L_{f}(s)=\int_{0}^{\infty} f(x) e^{-s x} d x
$$

provided that the integral exists for $\sigma>\sigma_{0}$ with some $\sigma_{0}$.

Let $0<\alpha, \beta \leq 1$. We define for $\Re s>1$ :

$$
\begin{aligned}
& \zeta(s ; \alpha, 0)=\sum_{n=0}^{\infty} \frac{1}{(n+\alpha)^{s}} \\
& \zeta(s ; 0, \beta)=\sum_{n=1}^{\infty} \frac{e^{2 \pi i n \beta}}{(n)^{s}} .
\end{aligned}
$$

The function $\zeta(s ; \alpha, 0)$ and $\zeta(s ; 0, \beta)$ call respectively the Hurwitz and Lerch zeta-functions. It is well-known that $\zeta(s ; \alpha, 0)$ has meromorphic continuation to the whole complex plane with simple pole $s=1$ with residue 1 , and $\zeta(s ; 0, \beta)$ is an entire function if $\beta \neq 1$.

The Lerch zeta-function produces two functions

$$
S(s ; \beta):=\sum_{n=1}^{\infty} \frac{\sin (2 \pi n \beta)}{n^{s}}, \quad C(s ; \beta):=\sum_{n=1}^{\infty} \frac{\cos (2 \pi n \beta)}{n^{s}},
$$

which are the entire functions if $\beta \neq 1$.

These functions are connected by the Hurwitz relation

$$
\begin{aligned}
& \zeta(s ; \alpha, 0)=\frac{2 \Gamma(1-s)}{(2 \pi)^{1-s}} \times \\
& \times\left(\sin \frac{\pi s}{2} \cdot C(a-1 s ; \alpha)+\cos \frac{2 \pi}{2} \cdot S(1-s ; \alpha)\right),
\end{aligned}
$$

$(\Re s<1)$.

Let us $\ell, q$ be positive integers, $\ell<q,(\ell, q)=1$, and let $\left(\ell_{1}, \ell_{2}\right)$ runs complete system of solutions of the congruence

$$
\ell_{1} \ell_{2} \equiv \ell \quad(\bmod q), 0<\ell_{1}, \ell_{2}<q .
$$

Define the function

$$
F(x)=\sum_{\substack{\ell_{1}, \ell_{2}=1 \\ \ell_{1} \ell_{2} \equiv \ell \\(\bmod q)}}^{q} \zeta\left(\frac{1}{2}+i x ; \frac{\ell_{1}}{q}, 0\right) \zeta\left(\frac{1}{2}-i x ; 0, \frac{\ell_{2}}{q}\right) .
$$


Consider the Laplace transform for $F(x)$

$$
L_{F}(s):=\int_{0}^{\infty} F(x) e^{-s x} d x .
$$

The main result of this paper is a proof the following statement.

Theorem 1. Let $\ell, q$ be the positive numbers, $(\ell, q)=1$. Then for the Laplace transform of the function $F(x)$ defined by (2), we have

$$
\begin{aligned}
L_{F}(s) & =\pi i\left(e^{\frac{i s}{2}}+e^{-\frac{i s}{2}}\right) c_{0}(\ell, q)+ \\
& +e^{-\frac{i s}{2}} \sum_{m, n=1}^{\infty} K(m,-n \ell ; q) \exp \left(-2 \pi i m n \ell^{-i s}\right)+\lambda_{\ell}(s),
\end{aligned}
$$

where $K(m,-n \ell ; q)$ is the Kloosterman sum and $\lambda_{\ell}(s)$ is analytic in the strip $|\Re s|<\pi$, and, for $|\Re s| \leq \pi-\varepsilon, \varepsilon>0$ is arbitrary small, the estimate $\lambda_{\ell}(s) \ll$ $(1+|s|)^{-1}$ holds. Moreover,

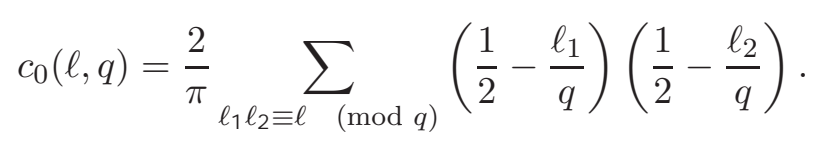

\section{Notation and Preliminary Results}

We will use the following notations. Denote by $\mathbb{C}$ the set of complex numbers; for $z \in \mathbb{C}$ we write $\exp (z)=e^{z}$. $\sum_{(\ell)}$ means the summation over all $\ell_{1}, \ell_{2}$ under condition $0<\ell_{1}, \ell_{2}<q, \ell_{1} \ell_{1} \equiv \ell(\bmod q)$. Symbol $\int_{(a)}$ always denotes integration an line $\Re z=a,-\infty<\Im z<+\infty$. The Vinogradov and Landau symbols (respectively, "«" and " $O$ ") are equivalent. $\Gamma(z)$ signifies the gammafunction. $\tau(n)$ is a divisor function.

We will apply some auxiliary lemmas.

Lemma 1. For $\frac{1}{2} \leq \Re s \leq 2,|\Im s| \geq 3$, we have

$$
\zeta\left(s ; \frac{\ell}{q}, 0\right), \zeta\left(s ; 0, \frac{\ell}{q}\right) \ll q^{c}(|\Im s|+3),(0<c<2),
$$

uniformly in $\ell, q,|\Im s|$. 
Lemma 2. The functions $e^{-y}$ and $\Gamma(s)$ form the Mellin pair, i.e. for $\Re s>0, b>0$,

$$
\Gamma(s)=\int_{0}^{\infty} e^{-x} x^{s-1} d x, \quad e^{-y}=\frac{1}{2 \pi i} \int_{b-i \infty}^{b+i \infty} \Gamma(s) y^{-s} d s
$$

The assertions of these lemmas are well-known (see, [7]).

Lemma 3. On the line $\Re s=\frac{1}{2}$ we have

$$
\begin{aligned}
& \sum_{(\ell)} \zeta\left(z ; \frac{\ell_{1}}{q}, 0\right) \zeta\left(1-z ; 0, \frac{\ell_{2}}{q}\right)= \\
& =\sum_{(\ell)} \zeta\left(\bar{z} ; \frac{\ell_{1}}{q}, 0\right) \zeta\left(1-\bar{z} ; 0, \frac{\ell_{2}}{q}\right) .
\end{aligned}
$$

Indeed, applying the Hurwitz relation and taking into account symmetry over $\ell_{1}, \ell_{2}$ of the left-hand side of equality (4) we obtain the assertion of Lemma 3 immediately.

We stand the following notations

$$
\begin{aligned}
& I_{1}(\ell, q):=\frac{e^{-\frac{i s}{2}}}{2} \int_{\left(\frac{1}{2}\right)} \sum_{(\ell)} \frac{\zeta\left(z ; \frac{\ell_{1}}{q}, 0\right) C\left(1-z ; \frac{\ell_{2}}{q}\right)}{\sin \frac{\pi z}{2}} e^{-i z\left(\frac{\pi}{2}-s\right)} d z, \\
& I_{2}(\ell, q):=\frac{e^{-\frac{i s}{2}}}{2 i} \int_{\left(\frac{1}{2}\right)} \sum_{(\ell)} \frac{\zeta\left(z ; \frac{\ell_{1}}{q}, 0\right) S\left(1-z ; \frac{\ell_{2}}{q}\right)}{\cos \frac{\pi z}{2}} e^{-i z\left(\frac{\pi}{2}-s\right)} d z, \\
& L_{F_{1}}(s):=\int_{0}^{\infty} \sum_{(\ell)}^{\infty} \zeta\left(\frac{1}{2}+i x ; \frac{\ell_{1}}{q}, 0\right) C\left(\frac{1}{2}-i x ; \frac{\ell_{2}}{q}\right) e^{-s x} d x, \\
& L_{F_{2}}(s):=\int_{0}^{\infty} \sum_{(\ell)} \zeta\left(\frac{1}{2}+i x ; \frac{\ell_{1}}{q}, 0\right) S\left(\frac{1}{2}-i x ; \frac{\ell_{2}}{q}\right) e^{-s x} d x .
\end{aligned}
$$

Lemma 4. There are exist the functions $\lambda_{\ell}^{(1)}(s), \lambda_{\ell}^{(2)}(s)$ that are analytic 
in the strip $|\Re s|<\pi$ and $\lambda_{\ell}^{(j)}(s) \ll(1+|s|)^{-1}, j=1,2$, such that

$$
\begin{aligned}
L_{F_{1}}(s) & =\frac{e^{\frac{i s}{2}}}{2} \int_{\left(\frac{1}{2}\right)} \sum_{(\ell)} \frac{\zeta\left(z ; \frac{\ell_{1}}{q}, 0\right) C\left(1-z ; \frac{\ell_{2}}{q}\right)}{\sin \frac{\pi z}{2}} e^{-i z\left(\frac{\pi}{2}-s\right)} d z+ \\
& +\lambda_{\ell}^{(1)}(s), \\
L_{F_{2}}(s) & =\frac{e^{\frac{i s}{2}}}{2 i} \int_{\left(\frac{1}{2}\right)} \sum_{(\ell)} \frac{\zeta\left(z ; \frac{\ell_{1}}{q}, 0\right) S\left(1-z ; \frac{\ell_{2}}{q}\right)}{\cos \frac{\pi z}{2}} e^{-i z\left(\frac{\pi}{2}-s\right)} d z+ \\
& +\lambda_{\ell}^{(2)}(s) .
\end{aligned}
$$

Proof. By the identity

$$
\begin{aligned}
1 & =\frac{\exp \left(\frac{\pi i}{4}-\frac{\pi x}{2}\right)}{\exp \left(\frac{\pi i}{4}-\frac{\pi x}{2}\right)-\exp \left(-\frac{\pi i}{4}+\frac{\pi x}{2}\right)}- \\
- & \frac{\exp \left(-\frac{\pi i}{4}+\frac{\pi x}{2}\right)}{\exp \left(\frac{\pi i}{4}-\frac{\pi x}{2}\right)-\exp \left(-\frac{\pi i}{4}+\frac{\pi x}{2}\right)},
\end{aligned}
$$

we have

$$
L_{F_{1}}(s)=I_{1}(\ell, q)+I_{11}(s)-I_{12}(s)
$$

where

$$
\begin{aligned}
I_{11}=I_{11}(s) & =\int_{0}^{\infty} \sum_{(\ell)} \frac{\zeta\left(\frac{1}{2}+i x ; \frac{\ell_{1}}{q}, 0\right) C\left(\frac{1}{2}-i x ; \frac{\ell_{2}}{q}\right)}{\exp \left(\frac{\pi i}{4}-\frac{\pi x}{2}\right)-\exp \left(-\frac{\pi i}{4}+\frac{\pi x}{2}\right)} e^{-x s} \times \\
& \times \exp \left(\frac{\pi i}{2}-\frac{\pi x}{2}\right) d x \\
I_{12}=I_{12}(s) & =\int_{0}^{\infty} \sum_{(\ell)} \frac{\zeta\left(\frac{1}{2}+i x ; \frac{\ell_{1}}{q}, 0\right) C\left(\frac{1}{2}-i x ; \frac{\ell_{2}}{q}\right)}{\exp \left(\frac{\pi i}{4}+\frac{\pi x}{2}\right)-\exp \left(-\frac{\pi i}{4}-\frac{\pi x}{2}\right)} e^{x s} \times \\
& \times \exp \left(-\frac{\pi i}{2}-\frac{\pi x}{2}\right) d x .
\end{aligned}
$$

The integrands in $I_{11}$ and $I_{12}$ involve two multipliers

$$
\zeta\left(\frac{1}{2}+i x ; \frac{\ell_{1}}{q}, 0\right) C\left(\frac{1}{2}-i x ; \frac{\ell_{2}}{q}\right)
$$


and

$$
e^{\mp x s} \frac{\exp \left(\mp \frac{\pi i}{4}-\frac{\pi x}{2}\right)}{\exp \left(\frac{\pi i}{4} \mp \frac{\pi x}{2}\right)-\exp \left(-\frac{\pi i}{4} \pm \frac{\pi x}{2}\right)},
$$

and moreover, the first multiplier increases as power of $x$ (precisely $\ll x^{\frac{1}{3}}$ ) and the second multiplier decreases if $x \rightarrow \infty$ as exponential function (precisely $\left.e^{-(\pi \pm s) x},|\Re s|<\pi\right)$.

So, the integrals $I_{11}$ and $I_{12}$ are convergent in the strip $|\Re s| \leq \pi-\varepsilon$ for any positive $\varepsilon \leq \frac{1}{2}$. Hence, we accomplish that $I_{11}-I_{12}$ defines analytic function in the strip $|\Re s|<\pi$.

Furthermore,

$$
\left|I_{1 j}\right| \leq\left|\int_{0}^{1}\right|+\left|\int_{1}^{\infty}\right| \ll 1+\left|\int_{1}^{\infty} G_{j}(x) e^{-x(\pi \pm s)} d x\right|, \quad(j=1,2),
$$

where

$$
\begin{aligned}
& G_{1}(x)=\sum_{(\ell)} \zeta\left(\frac{1}{2}+i x ; \frac{\ell_{1}}{q}, 0\right) C\left(\frac{1}{2}-i x ; \frac{\ell_{2}}{q}\right) \cdot\left(1-i e^{-\pi x}\right)^{-1}, \\
& G_{2}(x)=\sum_{(\ell)} \zeta\left(\frac{1}{2}+i x ; \frac{\ell_{1}}{q}, 0\right) C\left(\frac{1}{2}-i x ; \frac{\ell_{2}}{q}\right) \cdot\left(1+i e^{-\pi x}\right)^{-1} .
\end{aligned}
$$

Since, the function $C\left(z ; \frac{\ell_{2}}{q}\right)$ in the region $\frac{1}{2} \leq|\Re z| \leq 2$, $|\Im z| \geq 3$, has the same estimate as $\zeta\left(z ; 0, \frac{\ell_{2}}{q}\right)$, then the integral Cauchy formula gives that

$$
\left(\zeta\left(z ; \frac{\ell_{1}}{q}, 0\right)\right)^{\prime},\left(C\left(z ; \frac{\ell_{2}}{q}\right)\right)^{\prime} \ll q^{c}|\Im z| .
$$

And hence, computing the integrals $\int_{1}^{\infty} G_{j}(x) e^{-x(\pi \mp s)} d x, j=1,2$ by integrating by parts, we derive:

$$
\begin{aligned}
\int_{1}^{\infty} G_{j}(x) e^{-x(\pi \mp s)} d x & =\left.\frac{e^{-x(\pi \mp s)}}{-\pi \pm s} G_{j}(x)\right|_{1} ^{\infty}- \\
& -\int_{1}^{\infty} \frac{e^{-x(\pi \mp s)}}{-\pi \pm s} d G_{j}(x) \ll(1+|s|)^{-1} .
\end{aligned}
$$


Hence, we have that

$$
\lambda_{\ell}^{(1)}=I_{11}(s)-I_{12}(s)
$$

is analytic in the strip $|\Re(s)|<\pi$, and

$$
\lambda_{\ell}^{(1)} \ll(1+|s|)^{-1}
$$

From (9)-(13) the relation (7) and properties of $\lambda_{\ell}^{(1)}$ follow. In the same way we can prove the equality (8).

This completes the proof of Lemma 4 .

Corollary. In the strip $|\Re s|<\pi$ the following relation

$$
L_{F}(s)=I_{1}(\ell, q)+i I_{2}(\ell, q)+\lambda_{\ell}(s)
$$

where $\lambda_{\ell}(s)$ is analytic function, $\lambda_{\ell}(s) \ll(1+|s|)^{-1}$, holds.

\section{Main Result}

In this section we obtain the main result of our paper.

We start with calculation of the integrals $I_{j}(\ell, q), j=1,2$.

Note that the subintegral functions in $I_{1}(\ell, q)$ and $I_{2}(\ell, q)$ have simple poles at the points $s=0$ and $s=-1$, respectively.

Thus, denoting by $H_{1}(z, s)$ and $H_{2}(z, s)$ the subintegral functions in $I_{1}(\ell, q)$ and $I_{2}(\ell, q)$ (see, (5)), we obtain

$$
I_{1}(\ell, q)=\frac{e^{-\frac{i s}{2}}}{2}\left\{\int_{\left(-\frac{3}{2}\right)} H_{1}(z, s) d z+2 \pi i \underset{z=0}{\operatorname{ras}} H_{1}(z, s)\right\} .
$$

But we have

$$
\begin{aligned}
\underset{z=0}{\operatorname{res} H_{1}(z, s)} & =\frac{2}{\pi} \sum_{(\ell)} \zeta\left(0 ; \frac{\ell_{1}}{q}, 0\right) \cdot \sum_{m=1}^{\infty} \frac{\cos \left(2 \pi n \frac{\ell_{2}}{q}\right)}{n}= \\
& =\frac{2}{\pi} \sum_{(\ell)}\left(\frac{1}{2}-\frac{\ell_{1}}{q}\right)\left(\frac{1}{2}-\frac{\ell_{2}}{q}\right)=c_{0}\left(\ell_{q}\right),
\end{aligned}
$$


because the Hurwitz relation gives

$$
\zeta(0 ; u, 0)=\frac{1}{2}-u, \quad \zeta(1 ; 0, u)=\frac{1}{2}-u, \text { if } u \neq 1
$$

Hence

$$
I_{1}(\ell, q)=e^{-\frac{i s}{2}} \pi i c_{0}(\ell, q)+\frac{e^{-\frac{i s}{2}}}{2} \int_{\left(-\frac{3}{2}\right)} H_{1}(z, s) d z
$$

Further, by the Hurwitz relation, we deduce

$$
\int_{\left(-\frac{3}{2}\right)} H_{1}(z, s) d z=\sum_{(\ell)} \sum_{m, n=1}^{\infty}[\mathrm{L}+\mathrm{R}]=I_{1}^{(1)}(s)+I_{1}^{(2)}(s),
$$

where

$$
\begin{gathered}
\mathrm{L}=\cos \left(2 \pi n \frac{\ell_{1}}{q}\right) \cos \left(2 \pi m \frac{\ell_{2}}{q}\right) \int_{\left(-\frac{3}{2}\right)} \frac{2 \Gamma(1-z)}{(2 \pi m n)^{1-z}} e^{-i z\left(\frac{\pi}{2}-s\right)} d z, \\
\mathrm{R}=\sin \left(2 \pi \frac{n \ell_{1}}{q}\right) \cos \left(2 \pi \frac{m \ell_{2}}{q}\right) \int_{\left(-\frac{3}{2}\right)} \frac{2 \Gamma(1-z)}{(2 \pi m n)^{1-z}} \cdot \operatorname{ctg} \frac{\pi z}{2} \cdot e^{-i z\left(\frac{\pi}{2}-s\right)} d z,
\end{gathered}
$$

say.

Putting $1-z=w$ and applying Lemma 2, we find

$$
I_{1}^{(1)}(s)=\sum_{m, n=1}^{\infty} \sum_{(\ell)} \cos \left(2 \pi n \frac{\ell_{1}}{q}\right) \cos 2 \pi m \frac{\ell_{2}}{q} \exp \left(-2 \pi m n e^{-i s}\right) .
$$

For $I_{1}^{(2)}(s)$ we take into account that with every pairs $\left(\ell_{1}, \ell_{2}\right)$ involving in the sum $\sum_{(\ell)}$ as well as pair $\left(-\ell_{1},-\ell_{2}\right)$ involves. Hence,

$$
\sum_{\ell_{1} \ell_{2} \equiv 1} \sin \left(2 \pi n \frac{\ell_{1}}{q}\right) \cos \left(2 \pi m \frac{\ell_{2}}{q}\right)=0
$$

and, consequently, $I_{1}^{(2)}=0$. 
So, we obtain

$$
\begin{aligned}
I_{1}(\ell, q)=e^{-\frac{i s}{2}} \sum_{m, n=1}^{\infty} \sum_{(\ell)} & \cos \left(2 \pi m \frac{\ell_{1}}{q}\right) \times \\
& \times \cos \left(2 n \frac{\ell_{2}}{q}\right) \exp \left(-2 \pi i m n e^{-i s}\right)+ \\
& +\pi i e^{-\frac{i s}{2}} c_{0}(\ell, q) .
\end{aligned}
$$

Similarly as above, we obtain

$$
I_{2}(\ell, q)=\frac{e^{-\frac{i s}{2}}}{2 i}\left\{2 \pi i \operatorname{res}_{z=-1} H_{2}(z, s)+\int_{\left(-\frac{3}{2}\right)} H_{2}(z, s) d z\right\} .
$$

Obviously, that

$$
\underset{z=-1}{\text { res }} H_{2}(z, s)=-c_{0}(\ell, q) e^{-i\left(\frac{\pi}{2}-s\right)}=i e^{i s} c_{0}(\ell, q) .
$$

Moreover

$$
\begin{aligned}
\int_{\left(-\frac{3}{2}\right)} H_{2}(z, s) d z=\sum_{m, n=1}^{\infty} \sum_{(\ell)} & \left(\sin \left(2 \pi n \frac{\ell_{1}}{q}\right) \sin \left(2 \pi m \frac{\ell_{2}}{q}\right) \times\right. \\
\times & \int_{\left(-\frac{3}{2}\right)} \frac{2 \Gamma(1-z)}{(2 \pi m n)^{1-z}} e^{-i z\left(\frac{\pi}{2}-s\right)} d z+ \\
+ & \cos \left(2 \pi n \frac{\ell_{1}}{q}\right) \sin \left(2 \pi m \frac{\ell_{2}}{q}\right) \times \\
& \left.\times \int_{\left(-\frac{3}{2}\right)} \frac{2 \Gamma(1-z)}{(2 \pi m n)^{1-z}} e^{-i z\left(\frac{\pi}{2}-s\right)} d z\right)= \\
=\sum_{m, n=1}^{\infty} \sum_{(\ell)} \sin \left(2 \pi m \frac{\ell_{1}}{q}\right) & \sin \left(2 \pi n \frac{\ell_{2}}{q}\right) \exp \left(-2 \pi i m n e^{-i s}\right) .
\end{aligned}
$$

Thus, by combining (20)-(22), we have established the following representation

$$
\begin{aligned}
I_{2}(\ell, q) & =\pi e^{\frac{i s}{2}} c_{0}(\ell, q)+ \\
& +\frac{e^{-\frac{i s}{2}}}{2 i} \sum_{m, n=1}^{\infty} \sum_{(\ell)} \sin \left(2 \pi m \frac{\ell_{1}}{q}\right) \sin \left(2 \pi n \frac{\ell_{2}}{q}\right) \exp \left(-2 \pi i m n e^{-i s}\right) .
\end{aligned}
$$


Nowm from (19), (23) we have

$$
\begin{aligned}
L_{F}(s) & =\pi i\left(e^{-\frac{i s}{2}}+e^{\frac{i s}{2}}\right) c_{0}(\ell, q)+ \\
& +e^{-\frac{i s}{2}} \sum_{m, n=1}^{\infty} \sum_{(\ell)} \cos \left(2 \pi \frac{m \ell_{1}-n \ell_{2}}{q}\right) \exp \left(-2 \pi i m n e^{-i s}\right)+\lambda_{\ell}(s) .
\end{aligned}
$$

Since, $\left|\exp \left(-2 \pi i m n e^{-i s}\right)\right|=\exp (-2 \pi m n \sin (\pi-\Re s))$, we conclude that the series

$$
\sum_{m, n=1}^{\infty} \sum_{(\ell)} \cos \left(2 \pi \frac{m \ell_{1}-n \ell_{2}}{q}\right) \exp \left(-2 \pi i m n e^{-i s}\right)
$$

is an absolutely convergent series in $|\Re(s)| \leq \tau-\varepsilon$, and consequently $L_{F}(s)$ is an analytical function in this strip.

Further, we have

$$
\begin{aligned}
& \sum_{(\ell)} \cos \left(2 \pi \frac{m \ell_{1}-n \ell_{2}}{q}\right)= \\
& =\frac{1}{2} \sum_{(\ell)}\left(e^{2 \pi i \frac{m \ell_{1}-n \ell_{2}}{2}}+e^{2 \pi i \frac{n \ell_{2}-m \ell_{1}}{2}}\right)= \\
& =\sum_{\substack{\ell_{1}, \ell_{2} \\
\ell_{1} \ell_{2} \equiv \ell}}^{(\bmod q)}(\bmod q) \\
& e^{2 \pi i \frac{m \ell_{1}-n \ell_{2}}{q}}=K(m,-n \ell ; q),
\end{aligned}
$$

where $K(a, b ; q)$ is the classical Kloosterman sum, for which the bound of Weil[9]

$$
K(a, b ; q) \ll(\operatorname{gcd}(a, b, q))^{\frac{1}{2}} q^{\frac{1}{2}} \tau(q)
$$

is well-known.

Now, by the Corollary from Lemma 4 it follows that we completed the proof of main theorem.

\section{References}

[1] F.V. Atkinson, The mean-value of the Riemann zeta function, Acta Math., 81 (1949), 353-376.

[2] A. Balčiunas, A. Laurinčikas, The Laplace transform of Dirichlet Zfunction, Nonlinear Analysis: Modeling and Control, 17 (2)(2012), 122138. 
[3] A. Ivič, The Mellin transform of the square of Riemann's zeta-function, Int. J. Number Theory, 1 (1)(2005), 65-73.

[4] A. Ivič, The Laplace and Mellin transform of powers of the Riemann zetafunction, Int. J. Math. Anal., 1 (2)(2006), 113-140.

[5] M. Jutila, The Mellin transforms of the square of Riemanm zeta function, Periodica Mathematica Hungarica, 42 (2001), 179-190.

[6] M. Lukkarinen, The Mellin Transform of the Square of Riemann's Zeta Function and Atkinson's Formula, Ann. Acad. Scie. Fenn., Math. Diss. 140, Suomalainen Tiedeakatemia, Helsinki, 2005.

[7] K. Prahar, Primezahlverteilung, Springer-Verlag, Berlin, Götingen, Heidelberg, 1957.

[8] E. Titchmarsh, Theory of Functions, Oxf. Univ. Press, Oxford, 1939.

[9] A. Weil, On some exponential sums, Proc. Nat. Acad. Sci. U.S.A., 34 (1948), 204-207. 
